\title{
Non-Rigid Surface Matching Technique as a Tool in Scoliosis Deformation Modelling
}

\author{
Kim Siang, Ang \\ School of Engineering, University of Newcastle \\ angkimsiang@gmail.com
}

\begin{abstract}
We present a novel approach for deformation modelling and exploit it on scoliosis monitoring. Despite the extensive use of surface topographies to monitor scoliosis, shape change due to scoliosis deformities has never been satisfactorily resolved. A novel non-rigid surface matching algorithm with nine new parameters has been investigated and developed. This non-rigid matching algorithm has been trialled using models with predictable topographic deformation. There is evidence that surface deformities can be modelled. In addition, to demonstrate the capability of this new non-rigid matching algorithm in scoliosis modelling, we have performed experimental comparison with classical rigid matching algorithm using four different scoliosis data sets. The non-rigid matching algorithm returned r.m.s. values which were improved by at least 10\%. The experimental results are very promising, demonstrating that this new non-rigid matching algorithm is able to improve the precision and the accuracy of the matching. Analysis indicates that this new non-rigid matching algorithm has proven to be a very successful tool and is an improvement on the classical approach. Meanwhile the new parameters are able to delineate the possible spatial distribution of surface deformation.
\end{abstract}

\section{Background}

Scoliosis is a lateral or sideways curve in spine with concordant vertebral rotation that is apparent when viewing the spine from behind the person who has it. The most common treatment for scoliosis is based on "observation" in which the patient is monitored for any progression in the curvature. Observation can be done either using X-ray or non-invasive surface scanning. The use of radiographs (X-ray) to monitor the development of idiopathic scoliosis is the main laboratory examination method $[1,2]$. However, frequent X-ray screening may cause the patient exposure to high radiation. This is especially important for young women, because developing breast tissue has increased sensitivity to radiation, and repeated exposure in adolescence can increase the risk of breast cancer later on $[3,4,5]$.

The idea to develop non-invasive techniques to complement existing scoliosis measurement procedure (X-ray scanning) has been pursued for decades. The major advantage of non-invasive techniques compared to existing scoliosis measurement is it could be used more frequently allowing the practitioner to better document the changes of scoliosis. Laser scanning has been introduced in capturing the 3D back surface (surface topography) for scoliosis treatment and monitoring. Surface topography is an instant success for the patients and parents, in that they prefer not to be exposed to radiation and can believe that their complaint about deformities is being taken into account as well as the less easily accessible radiographic parameters. Furthermore surface topography is a valid measure of back surface deformities and can be used in place of radiography for monitoring patients, particularly in minor cases and in young children.

Since the derived 3D point cloud represent the object surface, the problem should be defined as surface matching problem. Based on the hypothesis that combining surface topography from two different epochs would produce better assessment result, we applied a least squares approach for aligning two back surface topographies to monitor the scoliosis deformation progression. Several techniques have been developed and reported for the purpose of identifying surface change $[6,7,8]$. However, deformation caused by scoliosis is a non-rigid change and classical rigid matching algorithm is not sufficient to transform and match the surfaces together. For this reason, we have studied and developed a new non-rigid surface matching algorithm with new parameters to overcome the drawbacks of the classical approach to tackle the surface matching problem in scoliosis monitoring.

\section{Introduction}

Surface matching, also referred to as registration without control points, describes an automated method used to find the parameters of a matching which minimises the separation between two 3D surfaces. In our research scenario, we are using laser scanned scoliosis data from the same patient obtained from different epochs to model the scoliotic change. In other words, the two surfaces are obtained from the same physical surface object at different epochs with an identical reference system. The surfaces are not $100 \%$ aligned because they are obtained in different epochs. In order to monitor the scoliosis deformation progression, a pair of surfaces will be matched together using the least squares approach to get the closest fit. Change parameters will be designed and employed to determine the deformations. 
Although the common affine transformation can improve the matching function compared with linear transformation, it does not mean the best transformation especially when applied on surfaces that are experiencing deformation. For this reason, we need to derive and concatenate extra parameters that are able to model the surface deformation. Extra parameters can be concatenated into single matrix by multiplying them together, in the order they are to occur. In the new nonrigid matching algorithm, we kept the three scale parameters to assess the natural growth of the patient and employed extra six shearing parameters to model the surface deformation. Object shears relative to a reference point varies depending on the shearing methods. Figure 1 depicts the various transformations for different shear parameters together with the definition of planes of motion. Forward bending of trunk is a motion in the sagittal (Y-Z) plane; lateral bending of the trunk is a motion in the coronal (X-Z) plane; and rotation of the trunk a motion in the transverse $(\mathrm{X}-\mathrm{Y})$ plane $[5]$.
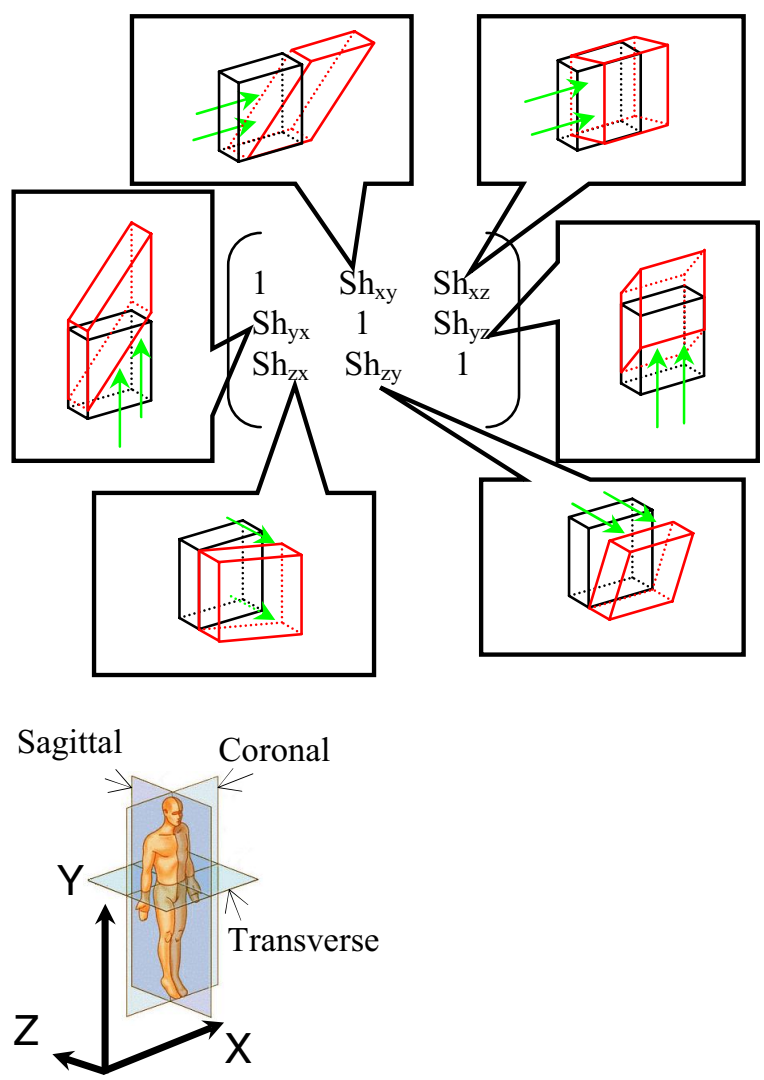

Figure 1: Illustralion of different shearing parameters and their transformation.

The deformations caused by shape changes are unknown for the matching algorithm. The algorithm minimises the sum of squares of the surface separation through mathematically moving one surface towards its closest fitting position to the other surface. It simultaneously models the deformations. The separations between the two surfaces are calculated from each point on Model Surface (S2) to a corresponding patch on the Reference Surface (S1). For an indication of the closeness of any two surfaces, root mean square (r.m.s.) and the value of residuals, V, derived from the alignment have been used. Successful modelling of all causes of change would reduce the r.m.s. and surface separation, V, to zero. The shearing parameters were then quantified with a deformities index. The deformities indices from the non-rigid matching were used to estimate the deformities due to scoliosis. The value of the r.m.s. of the residual surface differences at all measured points after mathematical surface topography alignment were used as a surface similarity index.

\section{Methodology}

This new non-rigid matching algorithm is based on a least squares solution as described in $[6,7,8]$. However, the matching function is replaced due to the drawbacks encountered in the classical approach. Instead of minimising the vertical difference as the matching function, the new non-rigid matching algorithm minimises the normal distance, $\mathrm{D}$, between the surfaces. We have studied both matching functions and found that minimising the normal distance is better than the classical matching function in term of the precision of the matching (defined by the residuals, $\mathrm{V}$, of the least squares) and the accuracy of the matching (which compares the r.m.s. errors). A similar study was trialled on small artificial data sets [9] in a comparison study of the matching function. The result is similar to ours. The non-rigid matching equation is given as:

$$
\left(\begin{array}{l}
X^{\prime} \\
Y^{\prime} \\
Z^{\prime}
\end{array}\right)=\left(\begin{array}{lcc}
1 & S h_{x y} & S^{x z} \\
S_{y x} & 1 & S^{y z} \\
S h_{z x} & S^{z y} & 1
\end{array}\right)\left(\begin{array}{ccc}
S_{x} & 0 & 0 \\
0 & S_{y} & 0 \\
0 & 0 & S_{z}
\end{array}\right)\left(\begin{array}{l}
X \\
Y \\
Z
\end{array}\right)
$$

Equation (1) can be written as:

$$
\begin{aligned}
& X^{\prime}=S_{x} \cdot X+S h_{x y} \cdot S_{y} \cdot Y+S_{x z} \cdot S_{z} \cdot Z \\
& Y^{\prime}=S_{y x} \cdot S_{x} \cdot X+S_{y} \cdot Y+S h_{y z} \cdot S_{z} \cdot Z \\
& Z^{\prime}=S h_{z x} \cdot S_{x} \cdot X+S h_{z y} \cdot S_{y} \cdot Y+S_{z} \cdot Z
\end{aligned}
$$

The normal distance of a point, $\left(\mathrm{x}_{1}, \mathrm{y}_{1}, \mathrm{z}_{1}\right)$, to a plane is given by the absolute value of:

$$
\mathrm{D}=\left|\mathrm{a}\left(\mathrm{x}_{1}\right)+\mathrm{b}\left(\mathrm{y}_{1}\right)+\mathrm{c}\left(\mathrm{z}_{1}\right)-\mathrm{d}\right| /\left(\mathrm{a}^{2}+\mathrm{b}^{2}+\mathrm{c}^{2}\right)^{1 / 2}
$$

where, coefficient $\mathrm{a}, \mathrm{b}$ and $\mathrm{c}$ are the component of vector $\mathrm{n}=(\mathrm{a}, \mathrm{b}, \mathrm{c})$ normal to the plane, $\mathrm{d}=-\mathrm{a}\left(\mathrm{x}_{0}\right)-\mathrm{b}\left(\mathrm{y}_{0}\right)$ - $\mathrm{c}\left(\mathrm{z}_{0}\right)$ with $\left(\mathrm{x}_{0}, \mathrm{y}_{0}, \mathrm{z}_{0}\right)$ a point on the plane, $\mathrm{S}_{\mathrm{x}}, \mathrm{S}_{\mathrm{y}}$ and $\mathrm{S}_{\mathrm{z}}$ are the scale parameters along the $\mathrm{x}, \mathrm{y}$, and $\mathrm{z}$ axis and $\mathrm{Sh}_{\mathrm{xy}}, \mathrm{Sh}_{\mathrm{xz}}, \mathrm{Sh}_{\mathrm{yx}}, \mathrm{Sh}_{\mathrm{yz}}, \mathrm{Sh}_{\mathrm{zx}}$ and $\mathrm{Sh}_{\mathrm{zy}}$ are the shear parameters.

Substitute Equation 2 into Equation 3, the following equation result: 


$$
\begin{aligned}
\mathrm{D}= & \mid \mathrm{a}\left(\mathrm{S}_{\mathrm{x}} \cdot \mathrm{X}+\mathrm{Sh}_{\mathrm{xy}} \cdot \mathrm{S}_{\mathrm{y}} \cdot \mathrm{Y}+\mathrm{Sh}_{\mathrm{xz}} \cdot \mathrm{S}_{\mathrm{Z}} \cdot \mathrm{Z}\right)+ \\
& \mathrm{b}\left(\mathrm{Sh}_{\mathrm{yx}} \cdot \mathrm{S}_{\mathrm{x}} \cdot \mathrm{X}+\mathrm{S}_{\mathrm{y}} \cdot \mathrm{Y}+\mathrm{Sh}_{\mathrm{yz}} \cdot \mathrm{S}_{\mathrm{z}} \cdot \mathrm{Z}\right)+ \\
& \mathrm{c}\left(\mathrm{Sh}_{\mathrm{zx}} \cdot \mathrm{S}_{\mathrm{x}} \cdot \mathrm{X}+\mathrm{Sh}_{\mathrm{z}} \cdot \mathrm{S}_{\mathrm{y}} \cdot \mathrm{Y}+\mathrm{S}_{\mathrm{z}} \cdot \mathrm{Z}\right)-\mathrm{d} \mid \\
& /\left(\mathrm{a}^{2}+\mathrm{b}^{2}+\mathrm{c}^{2}\right)^{1 / 2}
\end{aligned}
$$

A linearised observation equation relating $\mathrm{D}$ is setup and derived with respect to all the nine transformation parameters using Taylor expansion and keeping only the first order derivatives:

$$
\begin{aligned}
& \mathrm{D}^{*} \approx \mathrm{D}_{0}+\delta \mathrm{D} / \delta \mathrm{S}_{\mathrm{x}} \Delta \mathrm{S}_{\mathrm{x}}+\delta \mathrm{D} / \delta \mathrm{S}_{\mathrm{y}} \Delta \mathrm{S}_{\mathrm{y}}+\delta \mathrm{D} / \delta \mathrm{S}_{\mathrm{z}} \\
& \Delta \mathrm{S}_{\mathrm{z}}+\delta \mathrm{D} / \delta \mathrm{Sh}_{\mathrm{xy}} \Delta \mathrm{Sh}_{\mathrm{xy}}+\delta \mathrm{D} / \delta \mathrm{Sh}_{\mathrm{xz}} \Delta \mathrm{Sh}_{\mathrm{xz}}+\delta \mathrm{D} / \\
& \delta \mathrm{Shy}_{\mathrm{x}} \Delta \mathrm{Sh}_{\mathrm{yx}}+\delta \mathrm{D} / \delta \mathrm{Sh}_{\mathrm{yz}} \Delta \mathrm{Sh}_{\mathrm{yz}}+\delta \mathrm{D} / \delta \mathrm{Sh}_{\mathrm{zx}} \\
& \Delta \mathrm{Sh}_{\mathrm{zx}}+\delta \mathrm{D} / \delta \mathrm{Sh}_{\mathrm{zy}} \Delta \mathrm{Sh}_{\mathrm{zy}}
\end{aligned}
$$

where, $\mathrm{D}^{*}$ is the approximation of $\mathrm{D}, \mathrm{D}_{0}$ is the distance evaluated at the initial value of the parameters of the transformation, $\Delta \mathrm{S}_{\mathrm{x}}, \Delta \mathrm{S}_{\mathrm{y}}, \Delta \mathrm{S}_{\mathrm{z}}, \Delta \mathrm{Sh}_{\mathrm{xy}}, \Delta \mathrm{Sh}_{\mathrm{xz}}, \Delta \mathrm{Sh}_{\mathrm{yx}}$, $\Delta \mathrm{Sh}_{\mathrm{yz}}, \Delta \mathrm{Sh}_{\mathrm{zx}}$ and $\Delta \mathrm{Sh}_{\mathrm{zy}}$ are the corrections to initial values of the parameters, $\delta \mathrm{D} / \delta \mathrm{S}_{\mathrm{x}}, \delta \mathrm{D} / \delta \mathrm{S}_{\mathrm{y}}$, etc., are the partial derivatives with respect to the indicated unknows evaluated at the initial approximations.

Let $\mathrm{V}$ be the difference between $\mathrm{D}^{*}$ and $\mathrm{D}$ :

$$
\mathrm{D}=\mathrm{D}^{*}-\mathrm{V}
$$

Equation 5 can thus be written as:

$$
\begin{aligned}
& \mathrm{D}=\mathrm{D}_{0}+\delta \mathrm{D} / \delta \mathrm{S}_{\mathrm{x}} \Delta \mathrm{S}_{\mathrm{x}}+\delta \mathrm{D} / \delta \mathrm{S}_{\mathrm{y}} \Delta \mathrm{S}_{\mathrm{y}}+\delta \mathrm{D} / \delta \mathrm{S}_{\mathrm{z}} \\
& \Delta \mathrm{S}_{\mathrm{z}}+\delta \mathrm{D} / \delta \mathrm{Sh}_{\mathrm{xy}} \Delta \mathrm{Sh}_{\mathrm{xy}}+\delta \mathrm{D} / \delta \mathrm{Sh}_{\mathrm{xz}} \Delta \mathrm{Sh}_{\mathrm{xz}}+\delta \mathrm{D} / \\
& \delta \mathrm{Shy}_{\mathrm{x}} \Delta \mathrm{Sh}_{\mathrm{yx}}+\delta \mathrm{D} / \delta \mathrm{Sh}_{\mathrm{yz}} \Delta \mathrm{Sh}_{\mathrm{yz}}+\delta \mathrm{D} / \delta \mathrm{Sh}_{\mathrm{zx}} \\
& \Delta \mathrm{Sh}_{\mathrm{zx}}+\delta \mathrm{D} / \delta \mathrm{Sh}_{\mathrm{zy}} \Delta \mathrm{Sh}_{\mathrm{zy}}-\mathrm{V}
\end{aligned}
$$

With the model requirement that $\mathrm{D}=0$ and rearranging Equation 6, the following equation result:

$$
\begin{aligned}
& \mathrm{V}=\mathrm{D}_{0}+\delta \mathrm{D} / \delta \mathrm{S}_{\mathrm{x}} \Delta \mathrm{S}_{\mathrm{x}}+\delta \mathrm{D} / \delta \mathrm{S}_{\mathrm{y}} \Delta \mathrm{S}_{\mathrm{y}}+\delta \mathrm{D} / \delta \mathrm{S}_{\mathrm{z}} \\
& \Delta \mathrm{S}_{\mathrm{z}}+\delta \mathrm{D} / \delta \mathrm{Sh}_{\mathrm{xy}} \Delta \mathrm{Sh}_{\mathrm{xy}}+\delta \mathrm{D} / \delta \mathrm{Sh}_{\mathrm{xz}} \Delta \mathrm{Sh}_{\mathrm{xz}}+\delta \mathrm{D} / \\
& \delta \mathrm{Shy}_{\mathrm{x}} \Delta \mathrm{Sh}_{\mathrm{yx}}+\delta \mathrm{D} / \delta \mathrm{Sh}_{\mathrm{yz}} \Delta \mathrm{Sh}_{\mathrm{yz}}+\delta \mathrm{D} / \delta \mathrm{Sh}_{\mathrm{zx}} \\
& \Delta \mathrm{Sh}_{\mathrm{zx}}+\delta \mathrm{D} / \delta \mathrm{Sh}_{\mathrm{zy}} \Delta \mathrm{Sh}_{\mathrm{zy}}
\end{aligned}
$$

However, the numerator of $\mathrm{D}$ is an absolute value and is not definable at point equal to zero. As a result, signum function is used to differentiate $\mathrm{D}$. Let the variable $\eta$ be the expression inside the absolute value sign in Equation 4. Differentiating and simplifying Equation 7, the following partial derivative coefficients result:

$$
\begin{aligned}
& \delta \mathrm{D} / \delta \mathrm{S}_{\mathrm{x}}=\operatorname{sign}(\eta) \cdot\left(\mathrm{a} \cdot \mathrm{X}+\mathrm{b} \cdot \mathrm{Sh}_{\mathrm{yx}} \cdot \mathrm{X}+\mathrm{c} \cdot \mathrm{Sh}_{\mathrm{zx}} \cdot \mathrm{X}\right) \\
& /\left(a^{2}+b^{2}+c^{2}\right)^{1 / 2} \\
& \delta \mathrm{D} / \delta \mathrm{S}_{\mathrm{y}}=\operatorname{sign}(\eta) \cdot\left(\mathrm{a} \cdot \mathrm{Sh}_{\mathrm{xy}} \cdot \mathrm{Y}+\mathrm{b} \cdot \mathrm{Y}+\mathrm{c} \cdot \mathrm{Sh}_{\mathrm{zy}} \cdot \mathrm{Y}\right) \\
& /\left(a^{2}+b^{2}+c^{2}\right)^{1 / 2} \\
& \delta \mathrm{D} / \delta \mathrm{S}_{\mathrm{z}}=\operatorname{sign}(\eta) \cdot\left(\mathrm{a} \cdot \mathrm{Sh}_{\mathrm{xz}} \cdot \mathrm{Z}+\mathrm{b} \cdot \mathrm{Sh}_{\mathrm{yz}} \cdot \mathrm{Z}+\mathrm{c} \cdot \mathrm{Z}\right) \\
& /\left(a^{2}+b^{2}+c^{2}\right)^{1 / 2} \\
& \delta \mathrm{D} / \delta \mathrm{Sh}_{\mathrm{xy}}=\operatorname{sign}(\eta) \cdot\left(\mathrm{a} \cdot \mathrm{S}_{\mathrm{y}} \cdot \mathrm{Y}\right) /\left(\mathrm{a}^{2}+\mathrm{b}^{2}+\mathrm{c}^{2}\right)^{1 / 2} \\
& \delta \mathrm{D} / \delta \mathrm{Sh}_{\mathrm{xz}}=\operatorname{sign}(\eta) \cdot\left(\mathrm{a} \cdot \mathrm{S}_{\mathrm{z}} \cdot \mathrm{Z}\right) /\left(\mathrm{a}^{2}+\mathrm{b}^{2}+\mathrm{c}^{2}\right)^{1 / 2}
\end{aligned}
$$

$$
\begin{aligned}
& \delta \mathrm{D} / \delta \mathrm{Sh}_{\mathrm{yx}}=\operatorname{sign}(\eta) \cdot\left(\mathrm{b} \cdot \mathrm{S}_{\mathrm{x}} \cdot \mathrm{X}\right) /\left(\mathrm{a}^{2}+\mathrm{b}^{2}+\mathrm{c}^{2}\right)^{1 / 2} \\
& \delta \mathrm{D} / \delta \mathrm{Sh}_{\mathrm{yz}}=\operatorname{sign}(\eta) \cdot\left(\mathrm{b} \cdot \mathrm{S}_{\mathrm{z}} \cdot \mathrm{Z}\right) /\left(\mathrm{a}^{2}+\mathrm{b}^{2}+\mathrm{c}^{2}\right)^{1 / 2} \\
& \delta \mathrm{D} / \delta \mathrm{Sh}_{\mathrm{zx}}=\operatorname{sign}(\eta) \cdot\left(\mathrm{c} \cdot \mathrm{S}_{\mathrm{x}} \cdot \mathrm{X}\right) /\left(\mathrm{a}^{2}+\mathrm{b}^{2}+\mathrm{c}^{2}\right)^{1 / 2} \\
& \delta \mathrm{D} / \delta \mathrm{Sh}_{\mathrm{zy}}=\operatorname{sign}(\eta) \cdot\left(\mathrm{c} \cdot \mathrm{S}_{\mathrm{y}} \cdot \mathrm{Y}\right) /\left(\mathrm{a}^{2}+\mathrm{b}^{2}+\mathrm{c}^{2}\right)^{1 / 2}
\end{aligned}
$$

An observation equation of this form is written for each element on the S2, i.e. for every corresponding point-patch. Arranging the observation equations in matrix notation for least squares solution:

$$
\mathrm{V}_{(\mathrm{n}, 1)}=\mathrm{A}_{(\mathrm{n}, 9)} * \mathrm{X}_{(9,1)}+\mathrm{L}_{(\mathrm{n}, 1)}
$$

where, $V_{(n, 1)}$ is the vector of residuals, $A_{(n, 9)}$ is the coefficient matrix, $X_{(9,1)}$ is the vector of correction to the initial estimated unknown parameters and $\mathrm{L}_{(\mathrm{n}, 1)}$ is the vector of observation (the normal distance differences).

For each match, the program also provided various statistics such as number of iteration, standard deviation of the transformation parameters and mean surface distance separation. These statistics were used to help measure whether the match had proceeded satisfactorily. The quantitative output (e.g. numerical surface separation distances) was studied in conjunction with the graphical view (contour topographic map) from the matching to provide information about the nature of locations of the detected deformation.

\section{Results and Discussions}

Test cases using synthetic data were performed to demonstrate the ability of the matching algorithm to match a pair of surfaces. Two surfaces are created from the same object surface; S2 is transformed by known transformation parameters and S1 is retained in its original position and orientation. S2 is then transformed to match with $\mathrm{S} 1$. The tests were undertaken with different values of known parameters. These tests served the purpose of validating the matching algorithm by comparing the recovered transformation parameters with the known transformation parameters and the r.m.s. error. For briefness, Table 2 shows one of the matching results of the many test cases performed. An inspection shows that the algorithm is able to recover all the known parameters with standard of deviation of less than $0.005 \mathrm{~mm}$ and r.m.s. error less than $0.002 \mathrm{~mm}$. From the results obtained we can conclude that this new matching algorithm is validated and well-defined.

In addition, to demonstrate the capability of this new non-rigid matching algorithm in scoliosis monitoring, we have performed experimental comparisons with rigid matching algorithm with the help of four different scoliosis data sets. The matching results are shown in Table 3. In order to provide an overall picture of the matched results, a pair of scoliosis data set, B, transformed using these two algorithms is generated in contour topographic map and is presented in Figure 2. The numerical result of the transformation parameters is 
presented in Table 4. Meanwhile, the result obtained in this research was compared with the manual matching method defined in [10]. The final results were matched indicating this matching algorithm is feasible and favourable for scoliosis deformation monitoring. The comparison result is presented in Figure 3.

Table 2: Matching results from one of the test cases.

\begin{tabular}{llll}
\hline Parameters & $\begin{array}{c}\text { Known } \\
\text { Parameters }\end{array}$ & $\begin{array}{c}\text { Matching } \\
\text { Results }\end{array}$ & $\begin{array}{c}\text { Standard } \\
\text { Deviation }\end{array}$ \\
\hline $\mathrm{S}_{\mathrm{x}}$ & 1.2 & -1.198 & 0.0029 \\
$\mathrm{~S}_{\mathrm{y}}$ & 1.1 & -1.087 & 0.0013 \\
$\mathrm{~S}_{\mathrm{z}}$ & 1 & 1 & 0.0002 \\
$\mathrm{Sh}_{\mathrm{xy}}$ & 0.01 & -0.0108 & 0.0031 \\
$\mathrm{Sh}_{\mathrm{xz}}$ & 0 & -0.0001 & 0 \\
$\mathrm{Sh}_{\mathrm{yx}}$ & 0.02 & -0.0201 & 0.0040 \\
$\mathrm{Sh}_{\mathrm{yz}}$ & 0 & -0.0001 & 0.0002 \\
$\mathrm{Sh}_{\mathrm{zx}}$ & 0.01 & -0.0125 & 0.0024 \\
$\mathrm{Sh}_{\mathrm{zy}}$ & -0.01 & 0.0108 & 0.0021 \\
r.m.s. error & 0.00168 & & \\
Iteration & 5 & & \\
\hline
\end{tabular}

Table 3: Comparison of r.m.s. error between rigid and non-rigid matching algorithms from four different sets of scoliosis data.

\begin{tabular}{|c|c|c|c|}
\hline \multirow{2}{*}{$\begin{array}{l}\text { Scoliosis Data } \\
\text { (Point Density) }\end{array}$} & \multicolumn{2}{|c|}{ r.m.s. } & \multirow{2}{*}{$\begin{array}{c}\text { r.m.s. } \\
\text { Improvemen } \\
(\%)\end{array}$} \\
\hline & Rigid & $\begin{array}{l}\text { Non- } \\
\text { Rigid }\end{array}$ & \\
\hline $\mathrm{A}(<5000)$ & 2.538 & 2.160 & 15 \\
\hline $\mathrm{B}(>10000)$ & 1.670 & 1.492 & 11 \\
\hline $\begin{array}{c}\mathrm{C}(>10000 \mathrm{C}< \\
15000)\end{array}$ & 1.389 & 1.257 & 9.5 \\
\hline $\mathrm{D}(>15000)$ & 2.964 & 2.386 & 19.5 \\
\hline
\end{tabular}

In all cases, obviously there is an improvement in accuracy of at least $10 \%$ of the r.m.s. error by applying non-rigid matching. The maximum improvement of $\approx$ $20 \%$ is obtained for data with point density $>15000$. In addition to the expected accuracy, as observed from the contour topographic map in Figure 2, the displacement after the two surfaces matched using non-rigid matching algorithm has a better result (Figure 2(d), rectangle window) in non-deformed and less deformed areas compared with the rigid matching result. Figure 2(d) also clearly shows that a significant deformation pattern is present in one area that can be easily identified: on the left-hand side highlighted in a black box. The crucial finding is that the non-rigid matching has a significant improvement in matching the deformed areas (oval window in Figure 2(d)).

Note also that the new parameters can be directly used to explain the scoliosis progression. As observed from Table 4, there is significant shearing happening in $\mathrm{Y}$ and $\mathrm{Z}$ coordinates along $\mathrm{X}$ and $\mathrm{Y}$ axes (highlighted in bold). The shearing happened in transverse and sagittal plane with $0.0320 \mathrm{~mm}$ and $-0.0699 \mathrm{~mm}$ respectively. Special attention is required that the patient may experiencing a negative growth with $0.0409 \mathrm{~mm}$ in X. This negative growth may be caused by scoliotic deformation or other reason. Careful analysis needs to be carried out to identify and understand the cause. In conclusion, the transformation parameters could help in explaining the deformation magnitude and characterising the deformation progression caused by scoliosis. However, the real strength of this new algorithm lies in it potential for delineating deformation magnitude and direction which has not seen studied before especially in medical field.

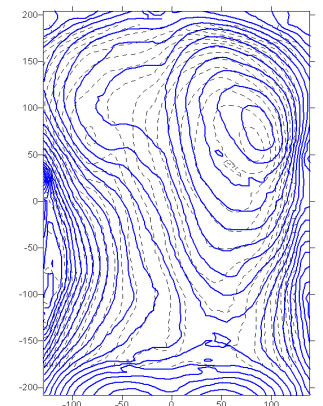

(a)

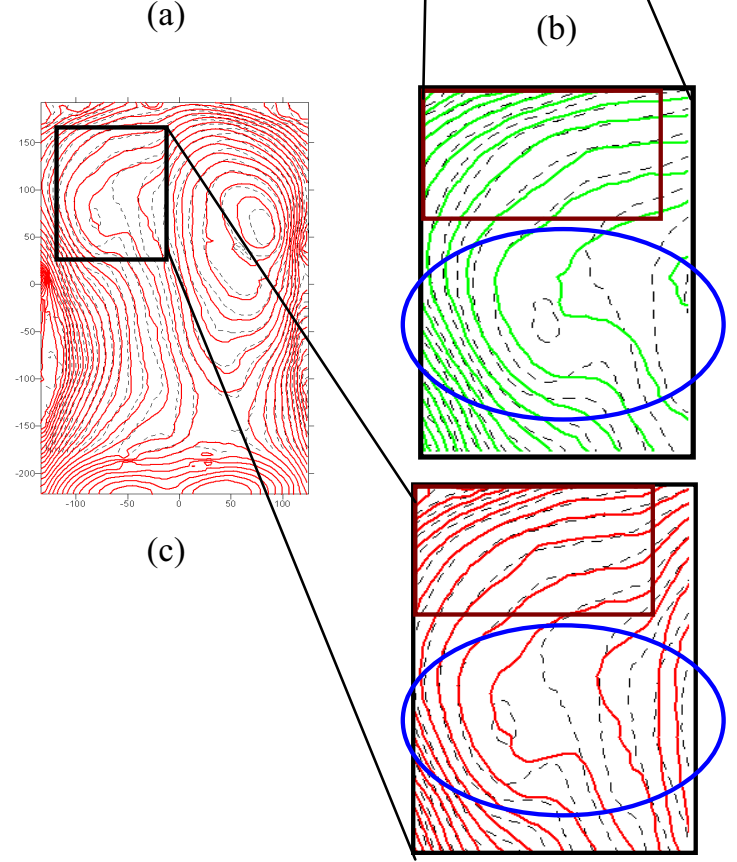

(d)
Figure 2: Contour topographic map of data set B, (a) before matching, (b) matched by rigid matching algorithm, (c) matched by non-rigid algorithm, (d) comparison of matched results for deformed (oval) and non-deformed (rectangle) areas between rigid and nonrigid algorithm. Solid lines represent surface B2 and dash lines are surface B1.

With the results obtained from the experiment, we can conclude that non-rigid matching is more suitable than rigid matching in matching surfaces that experience deformation. With the derivation of new non-rigid matching algorithm, we have achieved our major objectives. First, this new algorithm is able to model and ascertain the non-scoliosis change and 
scoliotic deformities. In our newly derived matching model, the scaling factor can be used to explain the dilation caused by natural growth and weight variation in $\mathrm{X}, \mathrm{Y}$, and $\mathrm{Z}$ direction respectively. Meanwhile, shearing parameters can be used to depict the deformation caused by scoliosis. Second, this new algorithm has improved the surface matching accuracy and overcome the drawbacks faced in the classical surface matching algorithm.

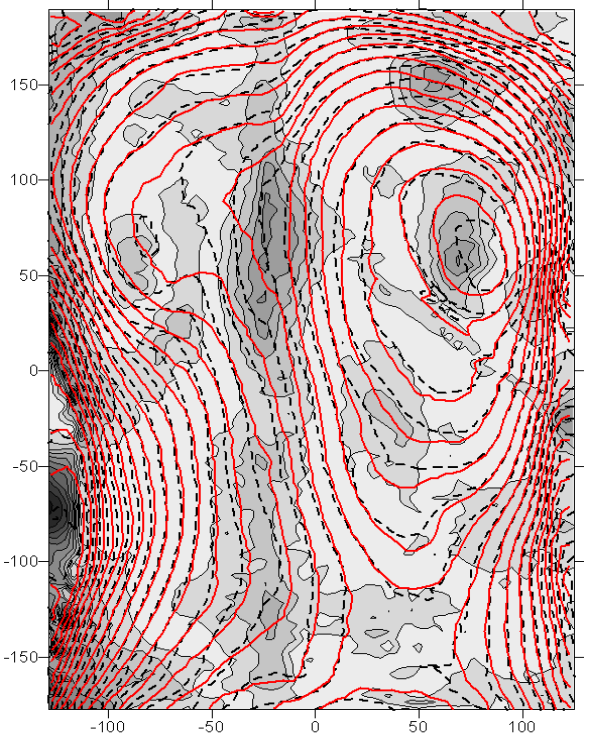

Figure 3: Comparison results of Difference Map (filled contour) defined in [3] with the results from non-rigid matching (normal and dashed lines). A very close deformities distribution pattern can be recognised on both results indicating this new non-rigid matching algorithm is able to detect surface deformities caused by scoliosis.

\section{Conclusions}

Although there has been active research in surface matching in different fields, surface matching in the medical field is still uncommon and limited research has been reported. Research in the medical field deserves special attention especially in scoliosis because it proved to be useful in detecting scoliosis deformation magnitude and progression. The 3D matching in this research is concerned with matching surface differences between epochs without the assistance of control point.

A new non-rigid algorithm has been developed which can match two surfaces and its parameters can be used to model the surface deformation. We have demonstrated this new algorithm is able to improve the surface matching and discards the drawbacks faced in classical surface matching algorithms. This new algorithm uses nine transformation parameters including six shear parameters which have not been used before to match the surfaces. The parameters we delineated are able to depict the surface deformation. This new non-rigid matching algorithm will give new insights to many problems in surface matching. The future development for this research perhaps is to develop a robust matching algorithm to eliminate outlier in the data.

Table 4: Parameters result from surface matching of scoliosis data set $\mathrm{B}$.

\begin{tabular}{|c|c|}
\hline Parameters & Results (mm) \\
\hline $\mathrm{S}_{\mathrm{x}}$ & -0.0409 \\
\hline $\mathrm{S}_{\mathrm{y}}$ & 0.0056 \\
\hline $\mathrm{S}_{\mathrm{z}}$ & -0.0007 \\
\hline $\mathrm{Sh}_{\mathrm{xy}}$ & -0.0105 \\
\hline $\mathrm{Sh}_{\mathrm{xz}}$ & 0.0020 \\
\hline $\mathbf{S h}_{\mathrm{yx}}$ & $\mathbf{0 . 0 3 2 0}$ \\
\hline $\mathrm{Sh}_{\mathrm{yz}}$ & 0.0136 \\
\hline $\mathrm{Sh}_{\mathrm{zx}}$ & 0.0197 \\
\hline $\mathbf{S h}_{\mathbf{z y}}$ & $\mathbf{- 0 . 0 6 9 9}$ \\
\hline
\end{tabular}

\section{References}

[1] C.J. Goldberg, M. Kaliszer, D.P. Moore, Surface Topography, Cobb Angles, and Cosmetic Change in Scoliosis, SPINE, 26 (4), (2001), E55-E63.

[2] X.C. Liu, J.G. Thometz, et al., Functional Classification of Patients with Idiopathic Scoliosis Assessed by the Quantec System, SPINE, 26 (11), (2001), 1274-1279.

[3] A.R. Levy, et al., Reducing the Lifetime Risk of Cancer from Spinal Radiographs among People with Adolescent Idiopathic Scoliosis, SPINE, 21 (13), (1996) 1540-1548.

[4] M.M. Doody, J.E. Lonstein, et al., Breast Cancer Mortality after Diagnostic Radiography: Findings from the U.S. Scoliosis Cohort Study, SPINE, 25 (16), (2000) 20522063.

[5] V. Pazos, F. Cheriet, et al., Accuracy Assessment of Human Trunk Surface 3D Reconstructions from an Optical Digitising System, Med. and Biol. Eng. and Comp., 43, (2005) 11-15.

[6] L.J. Pilgrim, Robust Estimation Applied to Surface Matching, ISPRS J. of Photogrammetric Eng. and Remote Sensing, 51, (1996) 243-257.

[7] H.L. Mitchell, and R.G. Chadwick, Mathematical Shape Matching as a Tool in Tooth Wear Assessment Development and Conduct, J. of Oral Rehabilitation, 25, (1998) 921-928.

[8] P. Miller, J. Mills, et al., A Robust Surface Matching Technique for Coastal Geohazard Assessment and Management, ISPRS J. of Photogrammetric and Remote Sensing, 63, (2008) 529-542.

[9] T. Schenk, A. Krupnik, and Y. Postolov, Comparative Study of Surface Matching Algorithms, In: XIXth Congress of the Int. Society for Photogrammetry and Remote Sensing, Vol. XXXIII, Part B4, (2000).

[10]D.C. Berg, D.L. Hill, et al., Using Three-Dimensional Difference Maps to Assess Changes in Scoliotic Deformities, Med. and Biol. Eng. and Comp., 40, (2002) 290-295. 